\title{
OPEN Modest changes in Spi1 dosage reveal the potential for altered microglial function as seen in Alzheimer's disease
}

\begin{abstract}
Ruth E. Jones ${ }^{1,2}$, Robert Andrews ${ }^{1,4}$, Peter Holmans ${ }^{3}$, Matthew Hill ${ }^{2}$ \& Philip R. Taylor ${ }^{1,2,4} \bowtie$
Genetic association studies have identified multiple variants at the SPI1 locus that modify risk and age of onset for Alzheimer's Disease (AD). Reports linking risk variants to gene expression suggest that variants denoting higher SPII expression are likely to have an earlier AD onset, and several other $A D$ risk genes contain PU.1 binding sites in the promoter region. Overall, this suggests the level of SPII may alter microglial phenotype potentially impacting AD. This study determined how the microglial transcriptome was altered following modest changes to Spi1 expression in primary mouse microglia. RNA-sequencing was performed on microglia with reduced or increased Spi1/PU.1 expression to provide an unbiased approach to determine transcriptomic changes affected by Spi1. In summary, a reduction in microglial Spi1 resulted in the dysregulation of transcripts encoding proteins involved in DNA replication pathways while an increased Spi1 results in an upregulation of genes associated with immune response pathways. Additionally, a subset of 194 Spi1 dose-sensitive genes was identified and pathway analysis suggests that several innate immune and interferon response pathways are impacted by the concentration of Spi1. Together these results suggest Spi1 levels can alter the microglial transcriptome and suggests interferon pathways may be altered in individuals with $A D$ related Spi1 risk SNPs.
\end{abstract}

Alzheimer's Disease (AD) is the most prevalent form of Dementia, affecting millions of people worldwide ${ }^{1}$. Studies investigating AD genetics and pathology have suggested immune gene networks may contribute to an increased risk of developing $\mathrm{AD}^{2,3}$. SPI1 encodes PU.1, a central transcription factor in microglial development and activation, and has a genome-wide significant genic association with AD in the IGAP GWAS (rs3740688 Odds Ratio 0.92 Meta $p=5.4 \times 10^{-13}$ ), comprising 35,274 Alzheimer's disease cases and 59,163 controls ${ }^{4}$. In addition, a 56-protein interaction network consisting of strongly enriched rare coding variants $\left(\mathrm{p}=1.08 \times 10^{-7}\right.$, and common variants with Late-Onset AD gene-wide significance $\left(\mathrm{p}=2.98 \times 10^{-7}\right)$ identified SPI1 as a central hub gene $^{5}$. Several studies have suggested SPI1/PU.1 levels impact on the microglial transcriptome, therefore affecting the phenotype of these cells. Several single-nucleotide polymorphisms (SNPs) associated with an increased risk of AD are thought to lie within the Spil gene locus ${ }^{3,6,7}$. The SNP variant rs $1057233^{\text {a }}$ is thought to result in a higher level of Spil expression and earlier age of AD onset ${ }^{7}$. Moreover, Spil is thought to influence the expression of other AD risk genes ${ }^{5,7,8}$.

The PU.1 transcription factor is essential for the survival and function of macrophages ${ }^{9-13}$ and is well conserved between humans and mice (> 85\% protein similarity, BLAST protein alignment (RefSeq ID's NP_003111.2 and NP_035485, respectively). In hematopoietic development low levels of PU.1 drives B-lymphocyte development whereas cells expressing high levels of PU.1 are committed to the myeloid lineage ${ }^{14-16}$. In development PU.1 levels are regulated to commit precursors to a macrophage or B-cell lineage ${ }^{17-23}$. In these early experiments PU.1 appeared to have dose-dependent transcriptional thresholds in foetal liver macrophages ${ }^{24}$. PU.1 also regulates expression of several key macrophage receptors such as CSF1R, CD11b and CD $45^{25-27}$. Moreover PU.1 interacts with other lineage-determining factors such as $\mathrm{C} / \mathrm{EPB} \alpha / \beta$ to alter the chromatin landscape resulting in a specialised macrophage epigenome ${ }^{28-30}$.

${ }^{1}$ Division of Infection and Immunity, Cardiff University, Cardiff, UK. ${ }^{2}$ UK Dementia Research Institute at Cardiff, Cardiff University, Hadyn Ellis Building, Maindy Road, Cardiff CF24 4HO, UK. ${ }^{3}$ Division of Psychological Medicine and Clinical Neurosciences, Cardiff University, Cardiff, UK. ${ }^{4}$ Systems Immunity Research Institute, Cardiff University, Cardiff, UK. ${ }^{\boxplus}$ email: TaylorPR@cardiff.ac.uk 
A

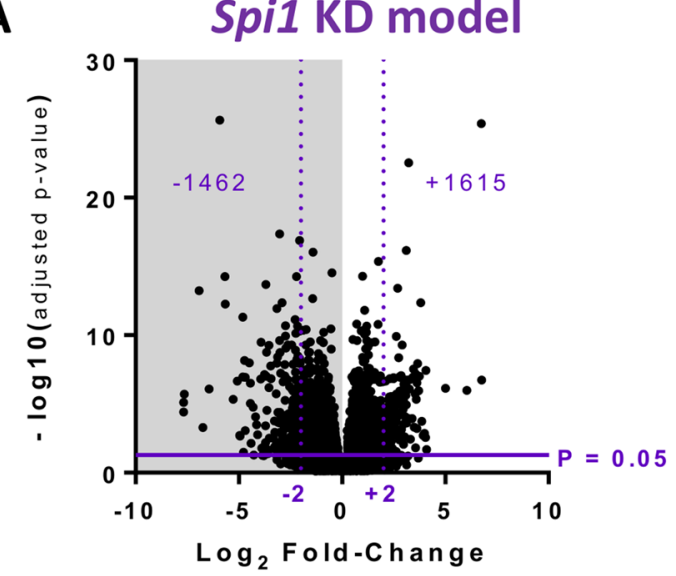

B

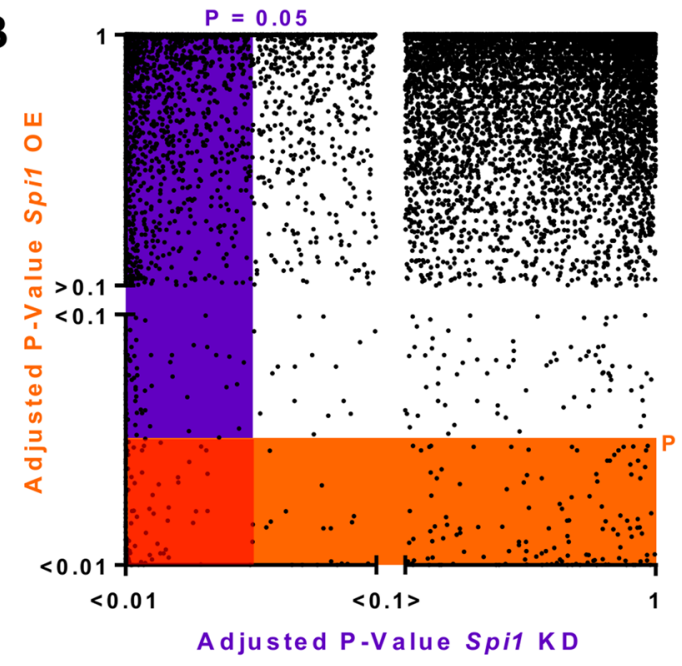

Spi1 OE model

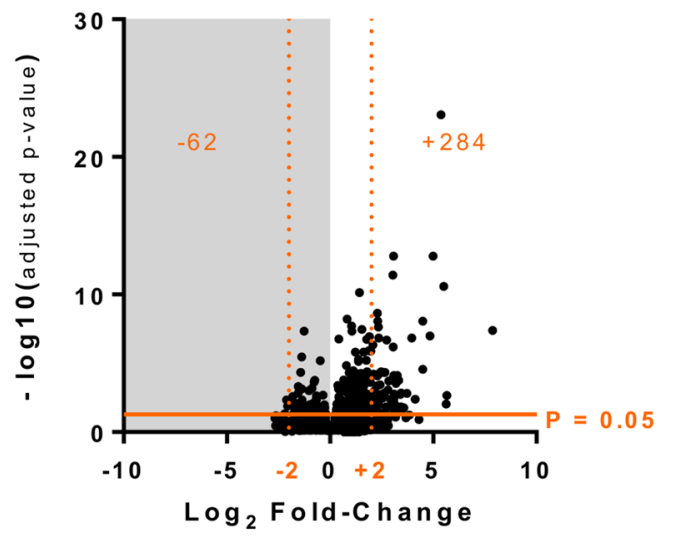

C

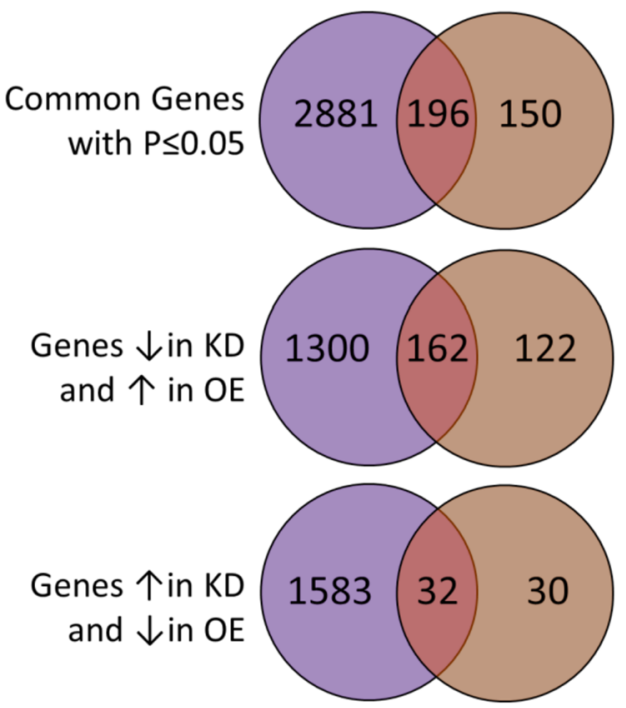

$\mathbf{E}$

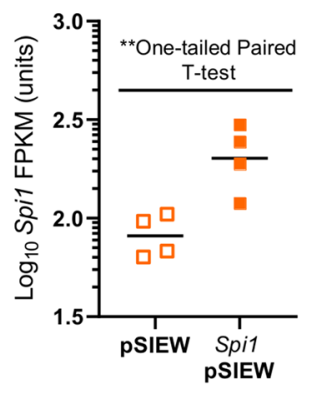

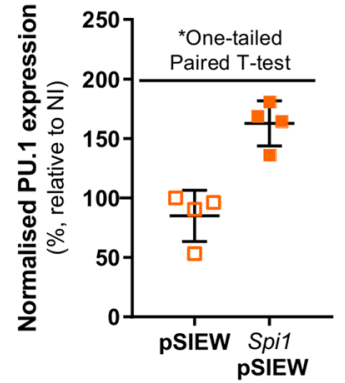
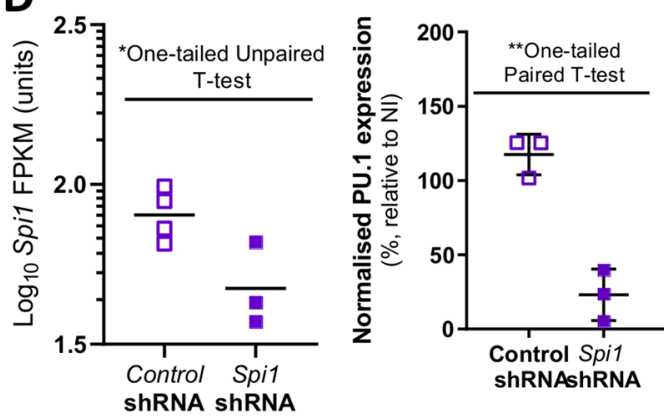

In both primary human microglia and the BV-2 mouse microglia cell line, reductions in PU.1 have resulted in changes to gene expression and a reduced phagocytic capacity ${ }^{7,31,32}$. Additionally, increased PU.1 expression in BV-2 cells resulted in increased zymosan phagocytosis, and amplified both ROS, NO and cytokine production after LPS stimulation ${ }^{32}$. Though the impact of altered Spil on the microglial transcriptome would ideally be studied in freshly isolated cells, such as those from a transgenic mouse model, at the time of this study no appropriate Spil over-expression transgenic mouse lines were available.

CSF1R inhibitors are a potential $\mathrm{AD}$ therapeutic and prevent $\mathrm{AD}$-associated microgliosis by blocking the CSF1R/PU.1 survival signalling pathway ${ }^{33-36}$ but the impact on peripheral macrophages has not been reported. Knowing how subtle changes in SPI1/PU.1 levels contribute to the microglial tissue resident subset transcriptome could allow more specific pathways could be targeted.

RNA-sequencing was used to assess changes to the microglial transcriptome following modest changes to Spi1 expression in microglia from primary mixed-glia cultures. Modest changes to PU.1 protein were desirable as they reflect the expression changes caused by the risk allele, and therefore the biology underlying AD. A moderate reduction in microglial Spi1 resulted in altered expression of cell cycle related genes while small Spi1 increases 
४Figure 1. Results of RNA-Seq experiment in primary mixed-glial cultures (A) Volcano plots summarising the distribution of genes in the Spi1 knock-down (purple) and Spil over-expression (orange). In the Spi1 knock-down dataset a 1462 genes were down-regulated (grey background) and 1615 up-regulated with a $p$ value of $\leq 0.05$ (solid lines), as indicated by the numbers on the graph. In the Spil over-expression dataset the majority of the genes, 284, were up-regulated and 62 genes had down-regulated expression. (B) Genes that were significantly changed in both the Spil knock down (KD, purple) and the Spil over expression (OE, orange) using a $p \leq 0.05$ threshold. A Plot of adjusted $p$ values from all genes in both datasets. Genes that were below the $p \leq 0.05$ threshold in the Spil knock-down dataset are highlighted in purple, those that were below the $p \leq 0.05$ cut-off in the Spil over-expression are within the orange bar. In the bottom left corner the red line surrounds the 196 genes that were significantly changed in both datasets. (C) Venn diagrams summarising the gene expression that were significantly changed $(p \leq 0.05)$ in either the Spil knock-down dataset (purple), the Spil overexpression dataset (orange) or changed in both datasets (red). Of interest were the genes with expression that appears to be sensitive to the Spil dose in microglia, namely the 194 genes that appear to be expressed relative to the dose of Spi1. (D) The $\log _{10}$ number of Spi1 mRNA fragments per Kilobase of transcript per Million mapped reads (FPKM) shows that Spil was lower in Spil shRNA infected microglia than in control shRNA infected microglia (One-tailed Unpaired T-test on the $\log _{10}$ transformed data, $p=0.0166, n=4$ for control shRNA and $\mathrm{n}=3$ for Spil shRNA). PU.1 protein expression was normalised to the non-infected (NI) samples in each experiment (as described in methods). Microglia infected with the Spi1 shRNA (solid purple) have reduced PU.1 expression compared to cells infected with a control shRNA (lined purple) (One-tailed Paired T-test, $p=0.0013, \mathrm{n}=3$ per group). (E) Spil mRNA expression (FPKM) was increased in the samples infected with the Spil over-expression construct (filled orange) compared to empty vector control samples (outline orange), (One-tailed Paired T-test on the $\log _{10}$ transformed data, $p=0.0013, \mathrm{n}=4$ per group). The Spil over-expression virus (Spi1 pSIEW) increased PU.1 protein expression in microglia by roughly half compared to cells infected with an empty vector control (lined orange). One-tailed Paired T-test, $p=0.0131, \mathrm{n}=4$ per group) (D-E) Each dot represents the value from one biological replicate, the means are indicated by the horizontal lines and the error bars display the standard deviation about the mean. All experiments were performed using mixed glia cultures from 8-week-old female C57BL/6J mice. Figures (A-C) were made in GraphPad PRISM 6 (version 3.07) and (D-E) in GraphPad PRISM 8 (version 8.4.3; both GraphPad Software, Inc.).

upregulated immune response genes. A subset of genes identified as Spi1 dose-sensitive highlighted a potential dose-dependent interferon driven immune response regulated by Spi1.

\section{Results}

RNA-sequencing data shows impact of Spi1 dose on microglia transcriptome. The effect of Spi1 shRNA knock down (KD) and Spi1 pSIEW over expression (OE) was assessed in flow cytometric sorted microglia after 11 days of lentivirus infection. Following Spi1 knock-down (Spi1 shRNA compared to control shRNA) 1615 genes were up- and 1462 down-regulated, using an adjusted $p$ value of $<0.05$ cut-off (Benjamini-Hochberg corrected for multiple testing). A proportion of down-regulated genes (230 genes) surpassed the $-2 \log _{2}$ foldchange cut-off (Fig. 1A). In the Spi1 over-expression dataset (Spi1 pSIEW compared to pSIEW) 284 genes had an increased expression, 62 were down-regulated $(p<0.05)$. In this comparison 71 up-regulated and 4 downregulated exceeded a $\log _{2}$ fold-change value of 2 . Principal component analysis (PCA, Supplementary Fig. 1) confirmed that samples within the same group had greater similarity, while the Spi1 pSIEW and Spi1 shRNA samples clustered separately.

The datasets with altered Spil expression were then compared to identify which subset of genes were likely affected by Spi1/PU.1 in a dose-dependent manner. This identified 196 genes which were significantly diminished in the Spil knock-down and upregulated in the Spil over-expression dataset (Adjusted $p$ value of $\leq 0.05$; Fig. 1B). When the direction of the gene fold-changes was compared 162 of these genes were lower in the dataset with lower Spil and higher in the Spil over-expression dataset and 32 genes where expression was increased following Spi1 knock-down and reduced in the Spil over-expression dataset (Fig. 1C). Therefore, 194 genes were classed as Spi1 dose-sensitive (Supplementary Table 1).

RNA-sequencing confirmed Spi1 fragments per Kilobase of transcript per Million mapped reads (FPKM) values were significantly lower in cultures infected with Spi1 shRNA as opposed to the control shRNA (Fig. 1D, One-Tailed Unpaired T-test on $\log _{10}$ data $p=0.0166$ ). Spi1 mRNA expression was increased in the Spil pSIEW samples compared to the control virus (Fig. 1E, One-Tailed Paired T-test on $\log _{10}$ data $p=0.0013$ ).

PU.1 protein was assessed in independent microglia samples (Fig. 1D-E) and was reduced by $\sim 70-80 \%$ in Spi1 shRNA samples compared to control shRNA infected microglia (Fig. 1D One-tailed Paired T-test, $p=0.0013$ and Supplementary Fig. 3B One-tailed Paired T-test, $p=0.001)$. Spil over-expression in microglia increased PU.1 protein expression by $\sim 70 \%$ compared to microglia infected with pSIEW control virus (One-tailed Paired T-test, $p=0.0131$ ). The values clearly cluster within each biological group and the means of Spil shRNA and Spi1 pSIEW microglia compared to the appropriate control viruses are disparate indicating PU.1 was altered by these Spil modulating viruses.

Gene ontology analysis in Spi1 knock-down and Spi1 over-expression datasets. Differentially expressed genes ( $p<0.05$ significance threshold) in the Spi1 KD and Spil OE datasets were separately assessed using DAVID (Database for Annotation, Visualization and Integrated Discovery, version 6.8). Figure 2 displays the Gene Ontology (GO) terms from the 20 most significantly enriched pathways in each dataset (corrected $p$ values $p<0.05, \mathrm{FDR}$ ). In primary microglia with a lower Spil expression the most significantly changed path- 
A

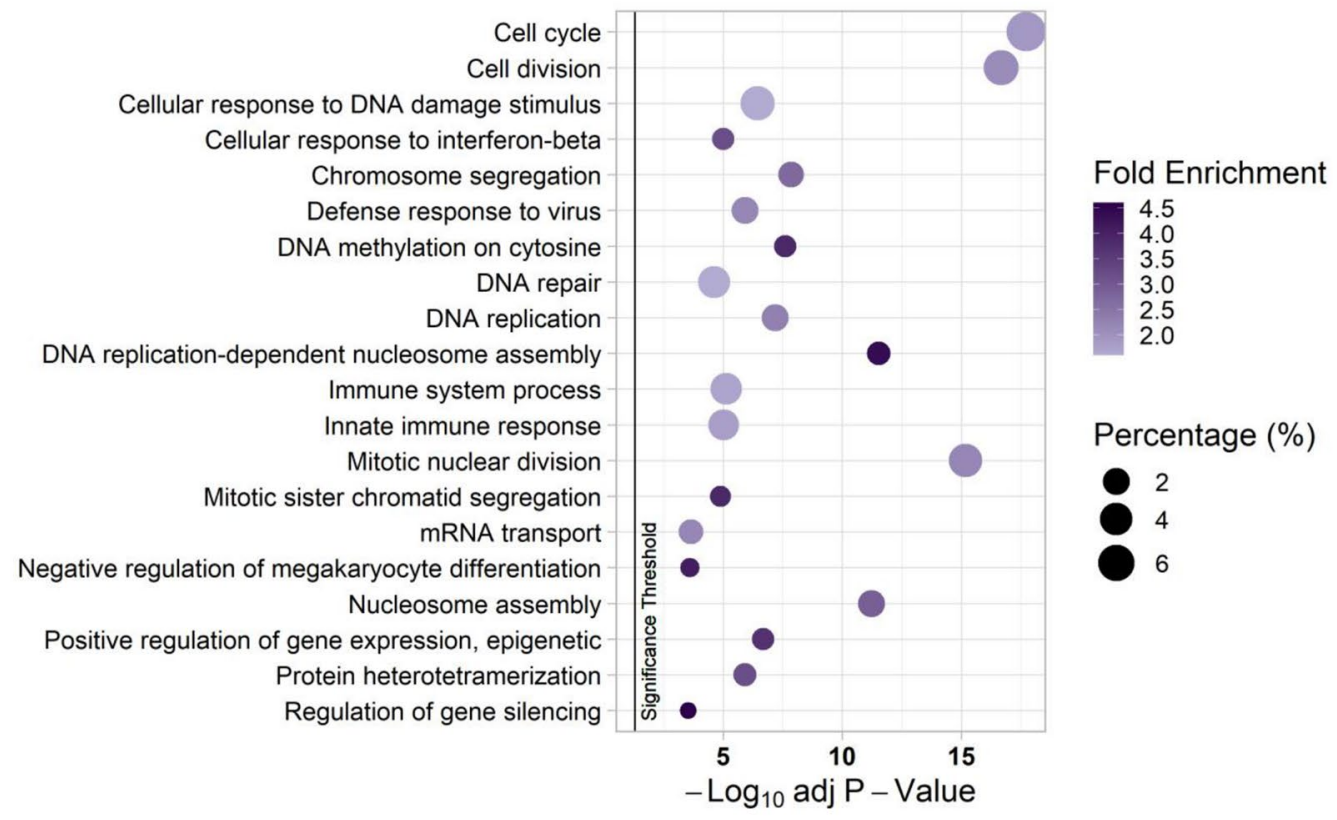

B

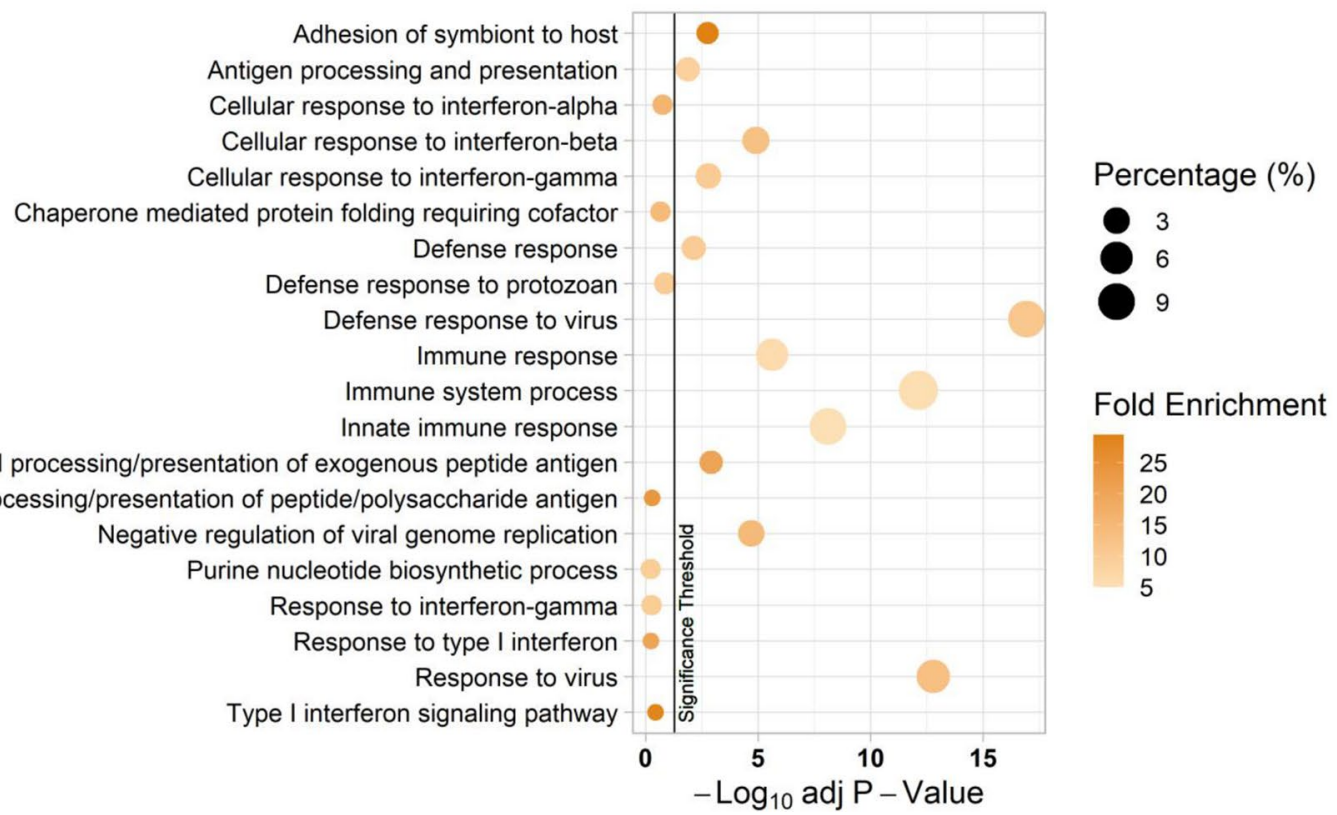

Figure 2. The 20 most siginificantly changed biological pathways in the Spi1 knock-down (A) and Spi1 over-expression (B) datasets assessed using DAVID. In these graphs the Benjamini-Hochberg adjusted $p$ value $\left(-\log _{10}\right)$ is displayed on the $\mathrm{x}$-axis, the Gene Ontology (GO) term listed on the $y$-axis, the percentage of the gene list in each cluster is denoted by the size of the bubble and the colour denotes the fold-change, where a darker colour indicates a stronger enrichment. The vertical black line indicates the $p$ value cut-off of 0.05 . Bubble plots were made using the 'tidyverse ${ }^{37}$ and 'ggpubr ${ }^{38}$ packages in $\mathrm{R}^{39,40}$.

ways were related to cell cycle and DNA repair (Fig. 2A). However, microglia with higher Spil (Fig. 2B) had an over representation of genes related to antigen presentation pathways, immune system processes and response to interferon.

Spi1 dose-sensitive subset highlight interferon response pathways. The absolute $\log _{2}$ foldchanges of 162 genes reduced by Spi1 knock-down and increased by Spil over-expression significantly correlated to each other (Fig. 3A) and the 32 genes that were increased by Spi1 knock-down and reduced by Spi1 over-expression (Fig. 3B) were also found to correlate (Two-tailed Spearman Rank Test approximate $p<0.0001$ and $r>0.8$ for both). This further supports the hypothesis that the expression of these genes is controlled in a Spil dose-sensitive manner. Collective analysis of the 194 Spil dose-sensitive gene list using GO terms through 
A 162 Genes $\downarrow$ in KD and $\uparrow$ in OE

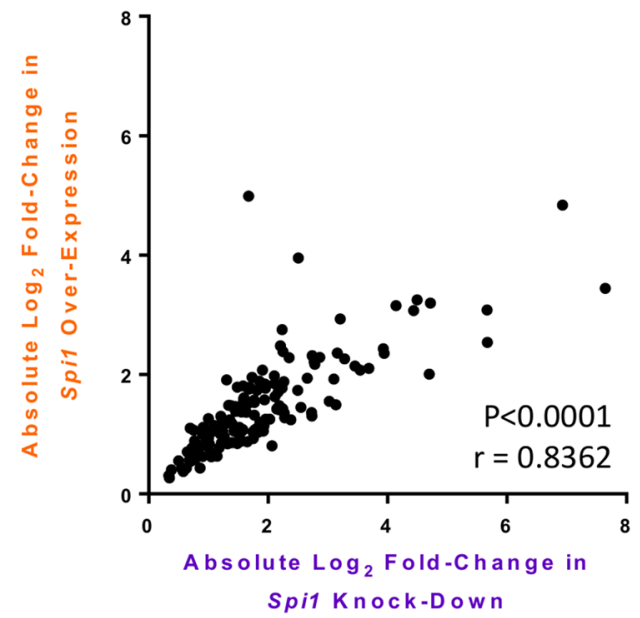

B $\quad 32$ Genes $\uparrow$ in KD and $\downarrow$ in OE

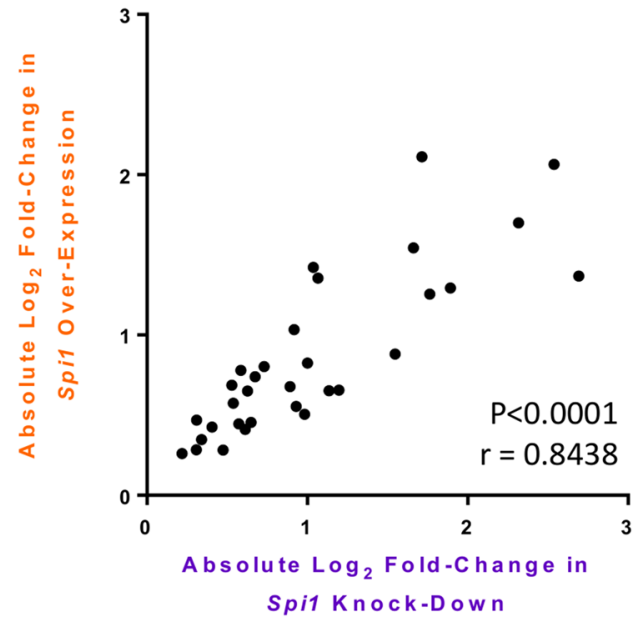

C

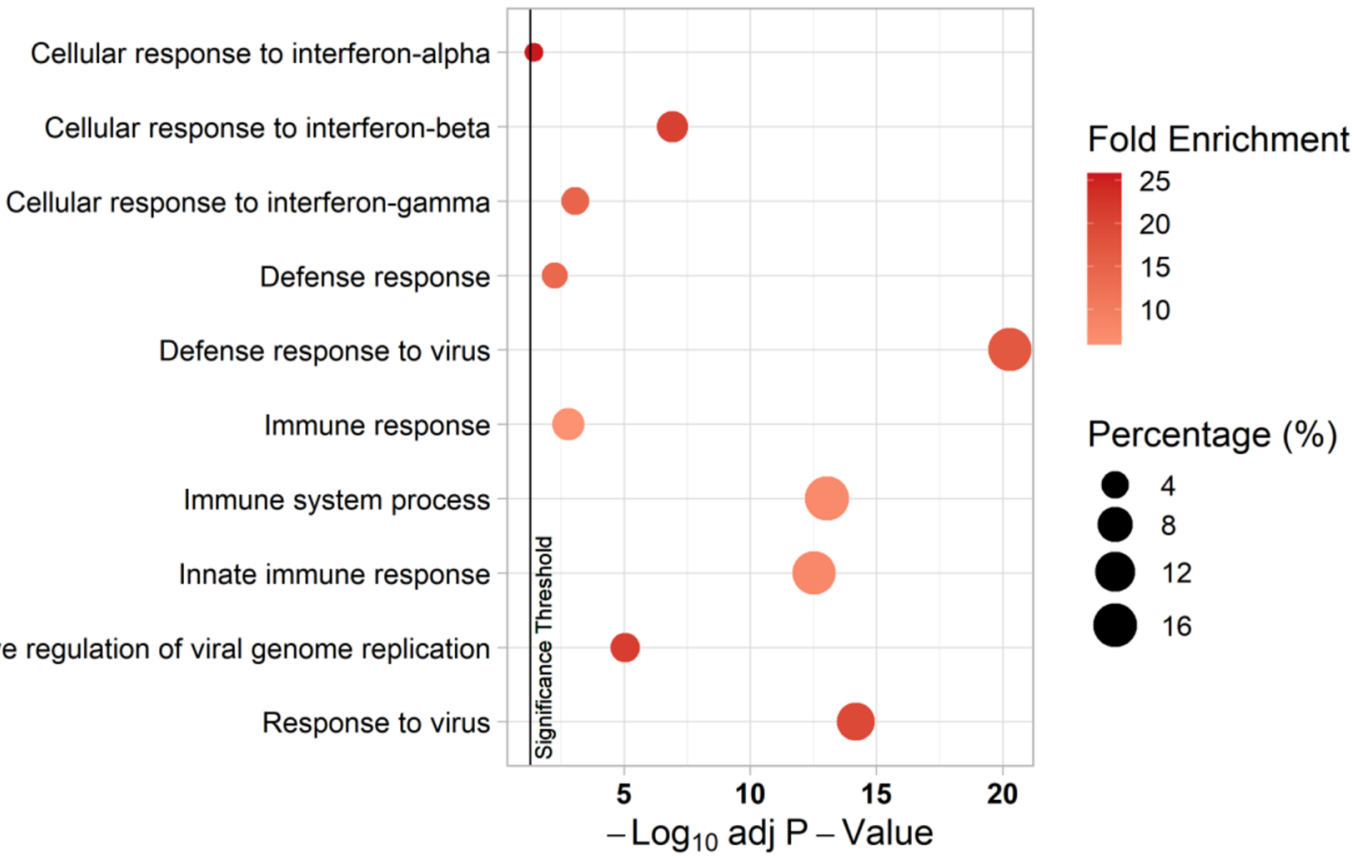

Figure 3. Absolute $\log _{2}$ fold-changes in Spi1 dose-sensitive genes. (A) Absolute $\log _{2}$ fold-change values were used the 162 genes where $p \leq 0.05$ with negative fold-change values in the Spil knock-down dataset (purple) and positive fold-change values in the Spil over-expression dataset (orange) were assessed separately from the 32 genes where $p \leq 0.05$ and Spil dose had the opposite effect on expression (B). Two-tailed Spearman Rank test identified significant correlations between the $\log _{2}$ fold-changes in both graphs (approximate $p<0.0001$ and $r>0.8$ ). (C) Top 10 most significantly altered "Biological Processes" according to the Benjamini-Hochberg adjusted $p$ value. The bubble size indicates the percentage of the gene list aligned to this pathway. The colour indicates the fold enrichment, which is the proportion of genes present in this list compared to the background gene expression. The vertical black line indicates the $p$ value threshold of 0.05 . Figures $(\mathbf{A}, \mathbf{B})$ were made in GraphPad PRISM 6 (version 3.07) and (C) using the 'tidyverse ${ }^{37}$ and 'ggpubr'38 packages in $\mathrm{R}^{39,40}$.

DAVID indicated an enrichment of immune response genes (Fig. 3C), particularly pathways linked to interferon and viral defence responses. These results suggest that a high microglial Spil expression may result in more responsive microglia that produce more interferon, though further work would be required to confirm this experimentally.

Several gene clusters implicate immune system responses. The data were assessed using hierarchical clustering of the FPKM expression values (Fig. 4). Six discrete clusters were identified, and gene lists were assessed using DAVID as before. Genes with an increased expression in microglia over-expressing Spi1 (Cluster 1) are enriched for pathways involving MHCII processing and presentation of antigens. The genes in Cluster 2 appear to be the most Spil sensitive, where expression was reduced in the Spil shRNA and increased in the Spil 
A

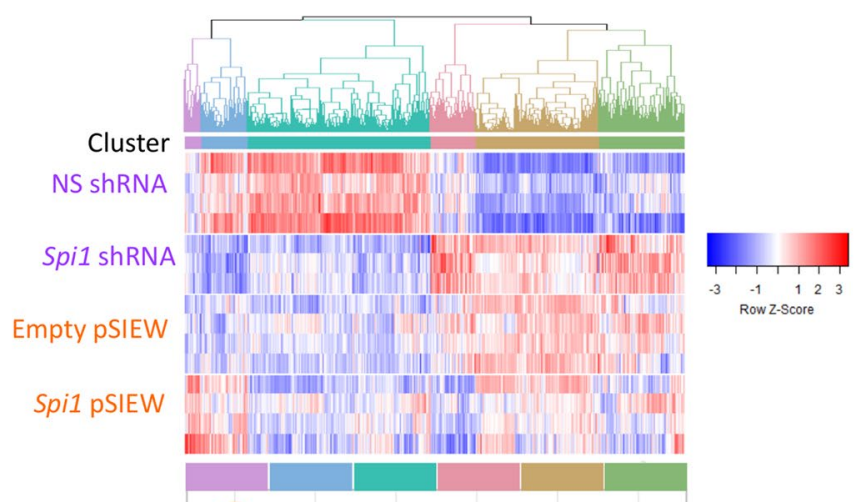

B

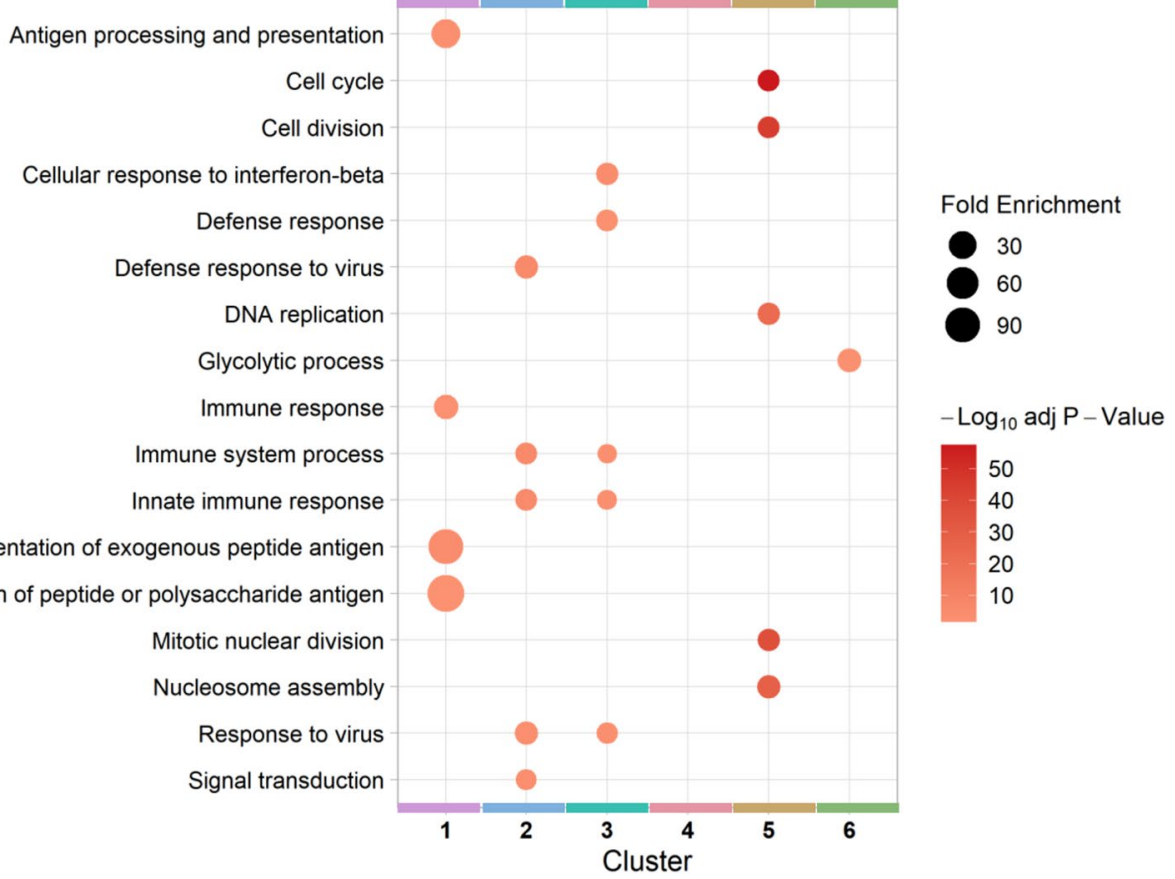

Figure 4. Hierarchical clustering analyses with the 20 most siginificantly changed biological pathways each cluster assessed using DAVID. (A) Hierarchical clustering of rows, where each row represents the scaled $\log _{10}$ FPKM values for each gene, with dendrogram higlighting cluster boundaries. The Pearson correlation was used to calculate the Z-Scores and UPGMA agglomeration methods were used. These 6 clusters were produced using the cutree function at a height of 1.59. (B) The gene lists were assessed using DAVID and a bubble plot created to highlight the top 5 most significatly enriched pathways ( $p$ value threshold of $<0.05$ ) in each gene cluster. Cluster 1 contains genes that had increased expression following a higher Spil expression and are mainly linked to immune responses and MHCII antigen presentation. Cluster 2 appears to contain more of the Spil dosesensitive genes and are enriched for viral/immune response signalling pathways. Genes in Cluster 3 had a lower expression in the Spil shRNA dataset and are related to immune response and interferon signalling pathways. Cluster 4 contained no significantly enriched pathways using the $p \leq 0.05$ threshold. Cluster 5 contains multiple genes related to cell cycle and DNA replication pathways, whose expression was increased in the Spil shRNA samples. The bubble size denotes the fold enrichment and the colour Benjamini-Hochberg adjusted $p$ values. Figures were made with the 'tidyverse ${ }^{37}$, 'gplots' ${ }^{22}$, 'plotly' ${ }^{43}$, 'dendextend' ${ }^{44}$ and 'colourspace ${ }^{45}$ packages in RStudio $^{39,40}$, code adapted from ${ }^{46,47}$ complete Markdown is available in Supplementary Information.

over-expression samples compared to relative controls. Most pathways associated with the genes in this cluster are related to the immune response including the response to viruses. Cluster 3 contained genes that were downregulated in the Spil shRNA samples compared to the controls and were related to interferon signalling and immune response pathways. Cluster 5 contains genes that had a higher expression in the Spil shRNA samples compared to the control shRNA samples. The GO TERMS associated with the genes in this cluster include those related to cell cycle and DNA replication pathways. Overall, this reinforces previous reports that reduced Spi1 seems to impact cell cycle which was not unexpected given the involvement of PU.1 in survival signalling ${ }^{11,41}$. A lowering of Spil appeared to reduce activation of immune response related pathways while increasing Spi1 expression seemed to have the opposite effect. 

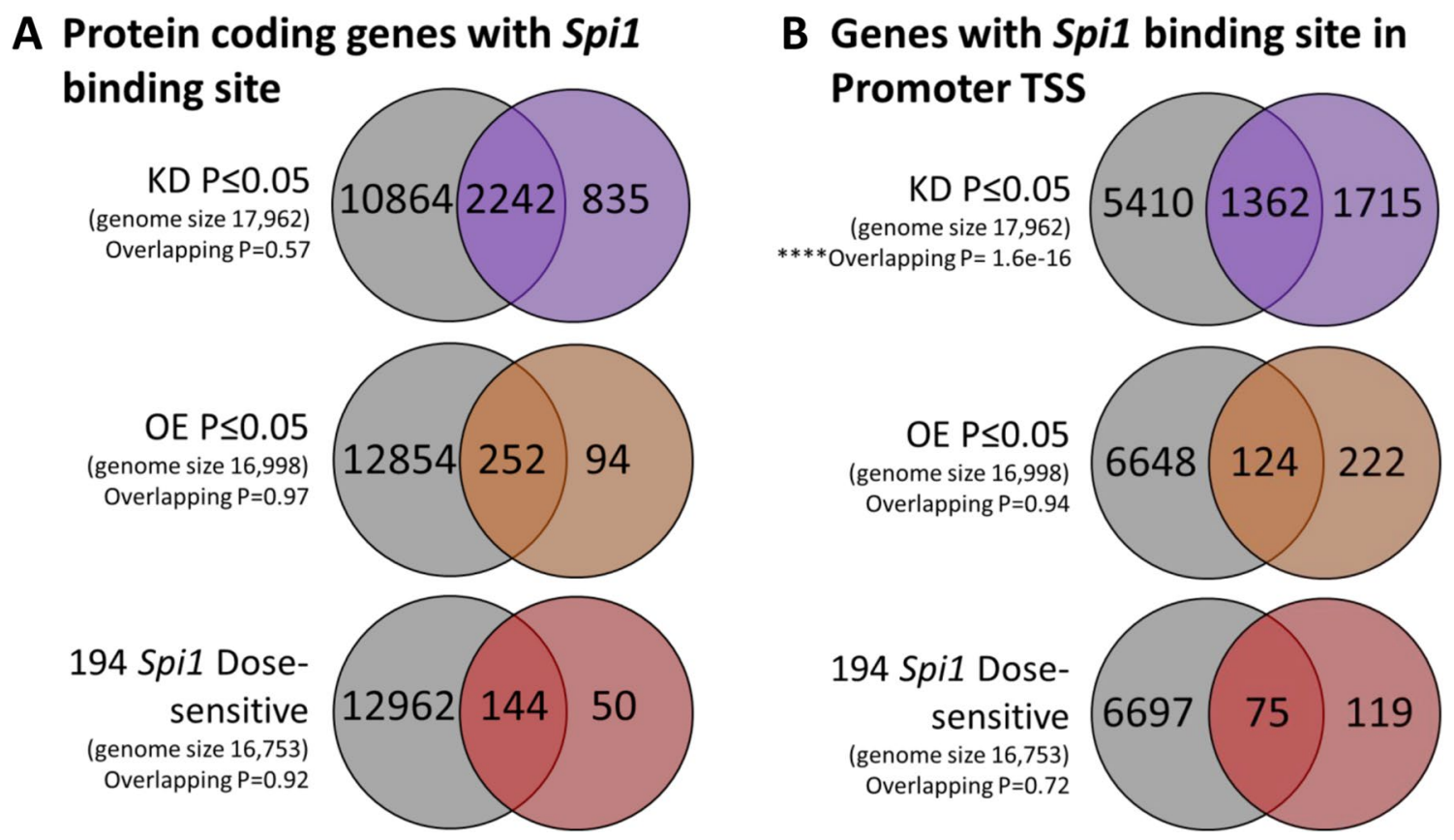

Figure 5. Comparison of Spi1 KD, Spi1 OE and 194 Spil Dose-sensitive genes to Spi1/PU.1 Chip-Seq dataset. Spil binding sites were determined from PU.1 Chip-Seq published data available online ${ }^{30}$ as described in methods. Fisher's exact tests were used to determine if there was a significant overlap between the datasets (A) includes the results from all protein coding genes with a Spil binding region (defined by Chip-seq in grey) that are also expressed in KD (purple), OE (orange) RNA-seq datasets or the Spil dose-sensitive genes (red). (B) The proportion of genes expressed in the RNA-seq datasets that contained a Spil binding site within the promoter region. The KD dataset contained a significant number of genes with a Spil binding sequence in the promoter (Fisher's exact test, overlapping $p$ value $=1.6 \times 10^{-16}$ ). No significant enrichment was noted in either the Spi1 $\mathrm{OE}$ or dose sensitive genes. Figures were made in PowerPoint based on results from 'GeneOverlap ${ }^{\text {' }}$ package in RStudio $^{39,40 .}$.

PU.1 dose-related transcriptomic differences were not limited by direct binding. Changes in gene expression after manipulation of PU.1 could arise from changes in direct DNA binding or via secondary effects. To identify possible direct targets of PU.1 that were differentially expressed, the proportion of protein coding genes that contained a PU.1 binding site sequence, especially within the promoter region, were assessed utilising a published ChIP-Sequencing (ChIP-Seq) dataset available online ${ }^{30}$. Figure 5 shows $\sim 70 \%$ of genes differentially expressed in the Spi1 RNA-Seq datasets contained a PU.1 binding site and therefore expression was potentially directly modified by Spi1. Moreover, 30-40\% of genes in Spi1 KD and Spi1 OE datasets had PU.1 binding sites within the promoter region suggesting their expression was directly altered by Spi1/PU.1. These proportions remained consistent even in the Spil dose-sensitive subset. Only differentially expressed genes in the Spi1 KD were enriched for PU.1 binding sites in the promoter region (Fisher's exact test, $p$ value of overlap $=1.6 \times 10^{-16}$ ). Given the proportion of overlapping genes was similar across all three test sets (Spi1 KD, Spi1 OE and Spi1 dose-sensitive) it seems Spi1 was not limited to directly regulating genes, but likely indirectly mediates expression of multiple other genes via secondary downstream processes.

Comparison to human AD risk genes. Protein network analysis suggest Spi1/PU.1 is one of several "hub" genes within a network of AD risk genes ${ }^{5}$, which was further supported by Cis-eQTL analyses in monocytes and macrophages ${ }^{7}$. The RNA-Sequencing profiles generated in this paper were compared to the International Genomics of Alzheimer's Project (IGAP) dataset, to investigate if there was an enrichment of AD genetic risk within the differential expressed sets of genes. Supplementary Table 2 denotes the gene sets used in this MAGMA analysis. In the Spil over-expression dataset a 21 gene set (Fig. 6), corresponding to a BenjaminiHochberg adjusted $p$ value of $\leq 1 \times 10^{-6}$ for differential expression, were enriched for AD genetic risk (adjusted $p$ value $=0.035$ ). The individual IGAP $p$ values can be viewed alongside the corresponding Spil over-expression data in Supplementary Table 3. This gene list (Fig. 6B) contained Ifit3, Oas1b and Oas2 which GO analysis linked to the interferon response pathway and $R n f 144 \mathrm{~b}$ and Treml 4 which are related to antigen presentation pathways, implicating the immune system response in $\mathrm{AD}$ pathology.

\section{Discussion}

Manipulation of Spi1 expression in primary mixed glia cultures were used to identify how microglial gene expression was altered following modest changes to Spi1/PU.1 (Fig. 1). Analysis of the RNA-sequencing datasets produced from these cultures have provided insight into how Spil dose affects the microglia transcriptome. Briefly Spi1 knock-down resulted in changes to gene expression of components of cell cycle checkpoint pathways while 

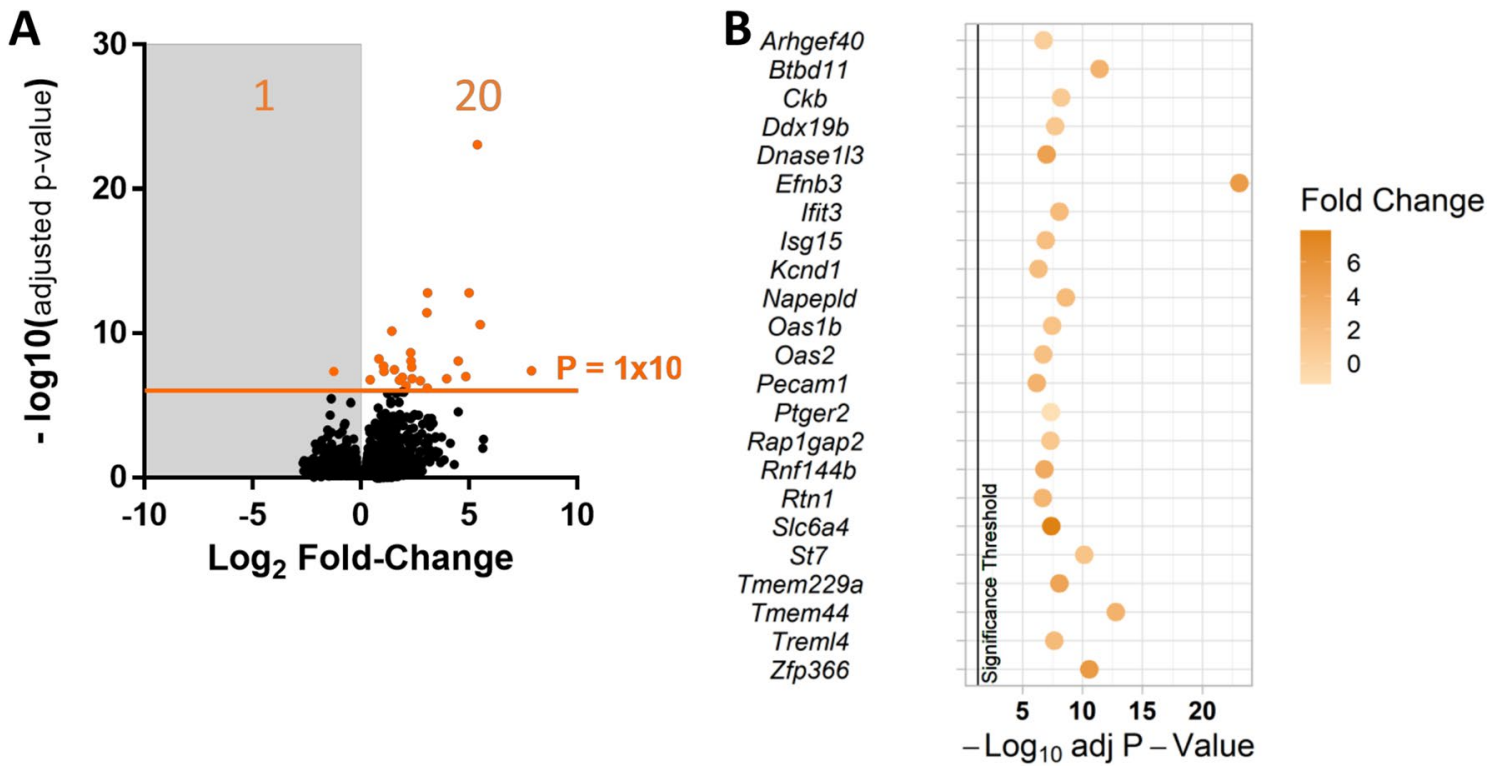

Figure 6. Summary of Spil over-expression genes that were significantly enriched in the IGAP dataset. (A) Volcano plot of the Spil over-expression gene expression changes relative to the empty vector control, highlighting the $p \leq 1 \times 10^{-6}$ threshold that was shown to be significantly enriched by MAGMA analysis in the IGAP dataset (MAGMA's empirical multiple testing corrected $p$ value 0.035 ). The adjusted $p$ value for this gene set was 0.0033 and an enrichment effect size of 0.51. (B) Bubble plot of the genes from the Spil over expression dataset that were enriched for AD genetic risk via MAGMA comparison to the human IGAP database, against the Benjamini-Hochberg adjusted $p$ values from the Spil over-expression RNA-sequencing data with the foldchange shown in colour. The vertical black line indicates the $p$ value threshold of 0.05 . Figure (A) was made in GraphPad PRISM 6 (version 3.07) and (B) using the 'tidyverse ${ }^{37}$ and 'ggpubr' ${ }^{38}$ packages in in RStudio ${ }^{39,40}$.

over-expression of Spi1 altered enriched pathways related to MHCII, interferon response and viral response (Figs. 3, 4). Comparing these RNA-Seq datasets to ChiP-Seq data suggests that approximately $70 \%$ of the differentially expressed genes were likely directly altered by Spi1 binding whereas the rest appeared to be altered via other mechanisms (Fig. 5).

Strengths of this experimental approach include assessing the bidirectional impact of Spil/PU.1 changes (Fig. 1) on the microglial transcriptome in an unbiased manner in primary cell cultures. We supplemented our cultures with TGF- $\beta$ which partially compensates for the loss of complex environmental cues microglia would normally receive. However, it does not fully replicate the in vivo context and there were no suitable transgenic mice available to study the impact of Spil dose on microglia in situ. The reduction in Spi1 FPKM values and PU.1 protein levels can be clearly seen in both microglia (Fig. 1D and Supplementary Fig. 3B) and in RAW264.7 cells (Supplementary Fig. 2). Figure 1E clearly shows that the Spi1 pSIEW plasmid results in increased Spi1 mRNA and PU.1 expression in microglia.

PU.1 has been previously linked to proliferation in macrophages. Bone-marrow derived Macrophages overexpressing PU.1 increased GM-CSF and M-CSF dependent proliferation and cell number, the opposite was seen in cells transfected with an anti-sense PU. $1^{9}$. However, there are some conflicting reports in the literature as to the role of PU.1 in microglia proliferation. In this model absolute cell numbers did not differ between Spi1 shRNA and control shRNA infected microglia, neither were there significant fold-change differences between normalised cell cycle data (Supplementary Fig. 3). In human microglia cultures an siRNA mediated reduction of PU.1 resulted in a lower cell number, disintegration and rounding of some cells but at the final timepoint viability appeared unaffected. This suggests some microglia could survive with reduced PU. $1^{41}$. Another similar study observed no reduction in microglia number, though PU.1 loss was measured at a culture level so the reduction could not be quantified on a single-cell level ${ }^{31}$. In summary, we did not observe significant changes in the proportion of cells in each phase of the cell cycle following a reduction in PU.1 (Supplementary Fig. 3), despite the differential expression of cell cycle associated genes within the Spil KD RNA-Seq dataset (Fig. 2).

PU.1 has been shown to bind the promoter of the Csf1-receptor (Csf1r) increasing expression of Csf1-receptor $^{49}$, which is highly linked to microglia survival and proliferation. In BV-2 cells a reduction in PU.1 also results in a reduced Csfir expression ${ }^{7}$ though no cell number/viability data was provided. It was recently shown that BV-2 cells with diminished PU.1 expression were more vulnerable to caspase-dependent cell death, while PU.1 over-expressing cells appear to have delayed onset of death. Baseline cell viability did not appear to be impacted by PU.1 modulation in these cells ${ }^{32}$. In the Spil RNA-seq datasets generated in this paper Csf1r expression was not significantly altered (Benjamini-Hochberg adjusted $p$ values 0.665 in $\mathrm{KD}$ and 0.999 in $\mathrm{OE}$ ), though the impact on Csf1r protein expression was not investigated here. Given that Csf1r inhibitors have been shown to prevent AD-related microgliosis and partially ameliorate disease pathology in vivo ${ }^{33-36}$ it would be interesting to see if Csf1R protein was affected in Spi1 shRNA infected microglia and if the reduction in PU.1 had a similar impact to Csf1r inhibitors on disease progress. 
Over-expression of Spi1 resulted in differentially expressed genes enriched for MHCII related pathways, interferon response pathways and response to virus pathways (Fig. 2B). Though there may be minor concerns that the significance of viral immune response pathways could be an unintended effect of using lentiviruses to manipulate Spil expression this is unlikely as comparisons were made relative to an almost identical control virus that lacked the Spi1 coding sequence. Additionally, this study identified a subset of 194 genes that appear to be Spil dose sensitive, linked to GO terms such as "Cellular response to interferon" and "Defence response to virus" (Fig. 3). Therefore, an increased level of Spi1 appears to cause transcription of genes associated with an inflammatory phenotype that might be relevant to AD genetic risk mechanisms (Fig. 6).

Previous work has identified that Spi1 is able to influence multiple gene networks in microglia ${ }^{5,7,31}$ and has been proposed as central to a network of AD risk genes that are conserved between species ${ }^{50}$. Basal cytokine expression was higher in BV-2 cells over-expressing PU.1, and was further potentiated by LPS stimulation compared to control samples ${ }^{32}$. Media taken from LPS treated PU.1 over-expressing BV-2 cells promoted a reactive astrocyte phenotype which was not observed in astrocytes treated with media from LPS treated BV-2 cells with reduced PU.1 expression ${ }^{32}$. Together these results suggest that PU.1 dose likely modulates the cytokine response in stimulated BV-2 microglia cells. In co-transfected NIH-3T3 fibroblasts PU.1 was required to bind either IFN Regulator Factor 4 (IRF-4) or IFN Consensus Binding Protein (ICSBP) for maximal induction of an IL-1 $\beta$ reporter assay ${ }^{51}$. Therefore, it is likely that increased Spi1/PU.1 expression levels in microglia may result in a more inflammatory microglial profile, potentially in conjunction with additional transcription factors. GO analysis of the Spi1 knock-down and over-expression datasets suggest that the level of Spi1 may impact microglial phenotype. Work in BV-2 cells suggests that reduced PU.1 results in a less reactive phenotype whereas PU.1 over expression primes cells for a more reactive response ${ }^{32}$. Further profiling is needed to ensure the pathway analysis matches the phenotype presented in this model or ideally in vivo.

Several gene-expression studies in AD model mice have highlighted Spi1 as a target of interest. Salih et al. identified a network of microglial genes expressed by amyloid-associated microglia, collated from five transgenic mouse lines, including Spi1, and 74 other genes that were identified as Spil dose-sensitive in this study ${ }^{52}$. These genes included Oas1b, Oas2, Ifit3 which were all highly differentially expressed in the Spi1 over-expression dataset (Fig. 6) and are linked to interferon signalling ${ }^{53}$. Sierskma et al. identified 18 genes that both overlapped with AD GWAS datasets and were maintained between young and old APP transgenic mice, which were predominantly expressed by microglia and appear to be regulated by $S p i 1^{50}$.

There are several reports in the literature that imply human and mouse microglia have phenotypic differences. For example, work in primary human microglia suggests that the responses to IFN- $\gamma$, TGF- $\beta 1$ and M-CSF may be species specific ${ }^{54}$. As this work was carried out in primary mouse cultures there could be concerns that the impact of reduced or increased Spi1/PU.1 might not be present in human biology.

However, studies investigating PU.1 in human microglia suggest there are shared Spi1/PU.1 dose dependent pathways in microglia. In addition to SPI1 binding sites being located near other AD risk genes or regulatory elements ${ }^{5,7,8}$, SPI1 risk alleles associated with a higher gene expression have been shown to lower the age of onset in $\mathrm{AD}^{7}$. Transcriptional profiles of microglia isolated from frozen human $\mathrm{AD}$ and control cortical tissue suggest an overall species difference in the disease associated signature, though an increased SPI1 expression was measured in $\mathrm{AD}$ samples compared to controls ${ }^{55}$ validating previous observations by others ${ }^{31}$.

In primary human microglia cultures siRNA mediated reductions in PU.1 resulted in a decrease in Amyloid- $\beta$ 1-42 peptide phagocytosis ${ }^{41}$, in a similar manner to observations in BV-2 cells ${ }^{7,32}$. Analysis of mixed microglia/ pericyte cultures where PU.1 was reduced via siRNA showed reduced expression of DAP12 and HLA-DR/DP/ $\mathrm{DQ}^{31}$. Moreover, similar gene expression changes to immune response and lipid metabolism genes were observed in BV-2 cells and human iPSC microglia following PU.1 siRNA knock-down ${ }^{32}$. Together these studies show that while the impact of SPI1 dose on microglia phenotype needs to be assessed in both species, some functional changes appear common to both human and mouse microglia.

In summary, disease relevant reductions in Spi1 highlighted dysregulation of genes involved in cell cycle checkpoint pathways. Modest increases in microglial Spil expression results in dysregulation of genes linked to immune response and interferon signalling, suggestive of a more pro-inflammatory microglial phenotype. This study highlights how relatively modest changes to Spi1/PU.1 expression alone can have a large impact on the microglial transcriptome of primary mixed-glial cultures, providing candidate pathways for future studies investigating Spil dependent processes and AD relevant biology.

\section{Methods}

Primary mixed glia cultures. Brains from 8-week-old C57BL/6 J mice (Charles River) were collected and transported in Hank's Balanced Salt Solution (HBSS without $\mathrm{Mg}^{2+}$ or $\mathrm{Ca}^{2+}$; Gibco) on ice. These culture experiments were repeated 10 times $(n=10), 4$ were used to generate RNA-Sequencing replicates and 6 for subsequent validation experiments. For most cultures three brains were sufficient per experiment (one 6 well-plate), though for some experiments more cells were required so cultures were scaled up accordingly. Brains were digested using the Neural Tissue Digest Kit-P in C-tubes as per manufactures directions (Miltenyi Biotec). Two brains were digested in each C-tube using program 37_ABDK on the GentleMACS OctoDissociator (Miltenyi Biotec) to produce a single cell suspension. Cell suspensions were passed through a $70 \mu \mathrm{M}$ strainer (Falcon) into a $50 \mathrm{~mL}$ tube and centrifuged at $300 \times g$ for $7 \mathrm{~min}$. The supernatant was aspirated and the pellet resuspended in $10 \mathrm{~mL}$ Dulbecco's Minimum Essential Media (DMEM containing $4.5 \mathrm{~g} / \mathrm{L}$ D-Glucose, GlutaMAX and supplemented with $15 \%(\mathrm{v} / \mathrm{v})$ heat-inactivated Foetal Bovine Serum (FBS) and $100 \mathrm{units} / \mathrm{mL}$ (v/v) Penicillin and $100 \mu \mathrm{g} / \mathrm{mL}$ Streptomycin (v/v); all Gibco) before centrifugation at $300 \times g$ for $7 \mathrm{~min}$. The supernatant was aspirated, and the pellet resuspended in $2 \mathrm{~mL}$ per brain of DMEM media containing $10 \mathrm{ng} / \mathrm{mL}$ recombinant murine M-CSF (Peprotech). Cell suspensions were then pooled as required and placed in a 6-well plate ( $2 \mathrm{~mL}$ per well) or 
Spil shRNA sequence $\left(5^{\prime} \rightarrow 3^{\prime}\right)$

GATGTGCTTCCCTTATCAAACCTCGAGGTTTGATAAGGGAAGCACATCTTTTT

Control shRNA sequence $\left(5^{\prime} \rightarrow 3^{\prime}\right)$

GTCTCGCTTGGGCGAGAGTAAGTAGTGAAGCCACAGATGTACTTACTCTCGCCCAAGCGAGACTTTTT

Table 1. shRNA sequences including termination sequence (bold).

$100 \mathrm{~mm} \times 20 \mathrm{~mm}$ (diameter $\times$ height) tissue culture plates, $12 \mathrm{~mL}$ per plate, and moved to a humid incubator with $95 \%$ air and $5 \% \mathrm{CO}_{2}$ overnight to allow the microglia to adhere.

Myelin was resuspended in the media (with $24 \mathrm{~h}$ of digest) by gentle plate agitation and the media containing the myelin debris was discarded. The cell monolayer was carefully washed with DMEM media. The wash media was then replaced with fresh DMEM media with $10 \mathrm{ng} / \mathrm{mL}$ M-CSF and this was again replaced two days later. On day 5 of culture the media was replaced with DMEM media containing $10 \mathrm{ng} / \mathrm{mL}$ M-CSF and $50 \mathrm{ng} / \mathrm{mL}$ recombinant mouse TGF- $\beta 1$ (eBioscience or Biolegend). TGF- $\beta$ supplementation was used to promote a more homeostatic phenotype in vitro ${ }^{56,57}$. Recombinant murine TGF- $\beta 1$ was purchased either from eBioscience or Biolegend, both of which were produced in a similar way. The Biolegend recombinant TGF- $\beta 1$ was diluted to a $100 \mu \mathrm{g} / \mathrm{mL}$ stock in an equal volume of sterile filtered 2\% BSA, $0.2 \mathrm{M}$ glycine in DPBS to match the eBioscience product.

From this point forward all culture media contained M-CSF and TGF- $\beta 1$ was replaced every two days. On day 10, these cultures were infected with Spi1 shRNA, control shRNA, Spi1 pSIEW or pSIEW lentivirus particles as appropriate. To achieve this the culture media was replaced with $3 \mathrm{~mL}$ fresh media and 100-300 $\mu \mathrm{L}$ of lentivirus was added to each plate. The volumes of Spil targeting and control viruses were kept the same within each experiment. After $6 \mathrm{~h}$ each plate received an additional $3 \mathrm{~mL}$ of culture media. The media on these cultures continued to be changed every 2 days, supplemented with M-CSF and TGF- $\beta 1$ as before.

All animal experiments were conducted in accordance with UK Home Office Guidelines and Animal [Scientific Procedures] Act 1986 which included full review and approval by the local ethical review board (Animal Welfare and Ethical Review Body, AWERB, of the Biological Standards Committee) and the granting of a UK Home Office Project Licence. The study was conducted in compliance with the ARRIVE guidelines.

Sample processing for RNA-sequencing. After 21 days the media was removed, set-aside, and the mixed glial cells harvested by incubating with $\sim 10 \mathrm{~mL}$ Accumax (Sigma) at $37^{\circ} \mathrm{C}$ for $10-20 \mathrm{~min}$. The monolayer was gently washed with DMEM media and any remaining attached cells were carefully removed using a plastic scraper (Greiner). The cell suspension was added back to the media and centrifuged at $300 \times g$ for 7 min. The supernatant was removed via pipetting and the cell pellet was resuspended in 0.5\% BSA in DPBS with a 1:1000 dilution of LIVE/DEAD near-IR staining solution for $30 \mathrm{~min}$ on ice per manufacturers direction (Molecular Probes).

Samples were centrifuged at $300 \times \mathrm{g}$ for $7 \mathrm{~min}$ and the supernatant aspirated via pipette. Each sample was resuspended in $500 \mu \mathrm{L}$ block solution $(4 \mu \mathrm{g} / \mathrm{mL}$ Rat anti-mouse Fc $\gamma \mathrm{RII} / \mathrm{III}$ (clone $2.4 \mathrm{G} 2$ ) and 5\% (v/v) filtered rabbit serum in $0.5 \% \mathrm{BSA}(\mathrm{w} / \mathrm{v}), 5 \mathrm{mM}$ EDTA in DPBS) and kept on ice for $10 \mathrm{~min}$. CD11b (Clone M1/70 PerCP-Cy5.5 conjugate, BD Biosciences final dilution $2 \mu \mathrm{g} / \mathrm{mL}$ ) and CD45 (Clone 30-F11 eFlour 450 conjugate, eBioscience final dilution $2 \mu \mathrm{g} / \mathrm{mL}$ ) antibodies were diluted to a $4 \mu \mathrm{g} / \mathrm{mL}$ concentration in $0.5 \% \mathrm{BSA}(\mathrm{w} / \mathrm{v}), 5 \mathrm{mM}$ EDTA in DPBS. $500 \mu \mathrm{L}$ of the antibody staining solution was added to each sample, to give a final antibody concentration of $2 \mu \mathrm{g} / \mathrm{mL}$, and the samples were incubated on ice for a further $15 \mathrm{~min}$. The tubes were then centrifuged at $300 \times g$ for $7 \mathrm{~min}$ and the supernatant removed.

The cells were resuspended in $1 \mathrm{~mL}$ of $0.5 \%$ BSA (w/v), 5 mM EDTA in DPBS and kept cool before undergoing fluorescent activated cell sorting on a FACS Aria III (BD Biosciences). Dead cells were excluded from the sort and microglia were selected using CD11b/CD45 double staining and GFP as a marker of infection. The sorted cells were then pelleted via centrifugation, the supernatant aspirated and the pellet lysed for RNA using the Mini RNeasy kit (Qiagen) per manufacturer's directions.

Lentivirus preparation. The shRNA vector construct and lentivirus production method has been described in $^{58}$ and sucrose-gradient purification in ${ }^{59}$. The insert sequences for the control shRNA and Spil shRNA can be seen in Table 1.

The over-expression plasmid used a spleen focus forming virus (SFFV) promoter to drive expression of murine Spi1 (Ensemble reference CCDS 16,425.1). An Internal Ribosome Entry Site (IRES) was used to initiate translation of a downstream eGFP reporter. Lentiviruses were purified by overlaying the virus containing media over a $20 \%$ sucrose solution before ultracentrifugation at $26,000 \mathrm{rpm}$ for $90 \mathrm{~min}$ at $4{ }^{\circ} \mathrm{C}$ in a SW28Ti swingingbucket ultracentrifuge rotor assembly in an Optima XPN-80 Ultracentrifuge (Beckman Optima Ultracentrifuge) and the viral pellet was resuspended in AimV media.

RNA-sequencing data analysis. RNA was isolated from cell sorted GFP + microglia using the RNeasy Mini kit (Qiagen) per manufacturer's instructions and eluted in nuclease free water. RNA integrity and concentration were assessed using the Agilent 2100 Bioanalyzer (Aligent). Complementary (cDNA) libraries were generated using the Truseq stranded total RNA with Ribo-Zero GOLD kit (Illumina). Paired end sequencing 
was then performed using the Illumina HiSeq 2500 sequencing platform to a read depth of between 30 and 40 million pairs.

The FASTQ files were processed with Trimmomatic ${ }^{60}$ to remove paired-end reads and quality was confirmed in FastQC using default parameters ${ }^{61}$. Following this sequence reads were mapped to the mm 10 genome (GRCm38) using the STAR pipeline ${ }^{62}$ and featureCounts was used to assign counts to transcripts ${ }^{63}$ with the GRCm38.84 Ensembl gene build GTF. The Ensembl FTP site was used to download the reference genome and $\mathrm{GTF}^{64}$. Differential gene expression was performed using the DESeq2 package ${ }^{65}$. Genes that were not significant after the differential expression analysis were discarded; significance was defined as an adjusted $p$ value (Padj) of $<0.05$ (Benjamini-Hochberg correction for multiple testing).

Assessing PU.1 promoter binding sites. A microglial PU.1 ChIP-sequencing (ChIP-seq) dataset (GSM1533906) isolated from C57BL/6 mice of a similar age $(8-9 \text { weeks })^{30}$ was accessed via the Cistrome database ${ }^{66}$. This dataset was then run through $\mathrm{HOMER}^{28}$ to generate an annotated peak file with genomic features. The promoter region was defined as 1000 base pairs upstream or 100 base pairs downstream of the transcription start site.

Duplicates were removed from the annotated ChIP-seq dataset and compared to gene discoveries from knockdown, over-expression and dose-sensitive Spil RNA-sequencing datasets. The background genome size was the number of genes detected in each RNA-Sequencing dataset defined as either all control and/or all experimental samples had a raw read count of $\geq 5$. In the Spil knock-down dataset 17,962 genes were detected and 16,998 genes in the Spil over-expression data. For the 194 Spil dose-sensitive genes a merged list of genes expressed in either the Spil knock-down or over-expression RNA-sequencing datasets was generated, and duplicates removed, to give a background gene number of 16,753 .

Finally, the ChIP-seq datasets were compared against their respective RNA-Seq dataset $(\operatorname{Padj} \leq 0.05)$ in $\mathrm{R}$ using the 'tidyverse' and 'GeneOverlap' packages. Here, a Fisher's exact test was utilised to confirm if there was any significant enrichment of PU.1 ChIP-seq binding sites in genes expressed in Spil knock-down, over-expression or dose-sensitive datasets.

Gene ontology analysis. Enriched gene Ontology (GO) terms were identified using the Database for Annotation, Visualization and Integrated Discovery (DAVID, version 6.8) and compared against a background of genes expressed in at least one Spi1 RNA-seq dataset (16,998 genes). Gene expression in the Spi1 RNA-seq datasets was defined as having a raw read count $\geq 5$ in all control and/or all experimental samples. The most significantly altered terms from the GOTERM_BP_DIRECT list, henceforth called Biological Process GO Terms, were downloaded for subsequent analysis. Bubble plots were generated in R using the 'ggplot2' package, and an adjusted $p$ value cut-off of 0.05 as indicated on each plot. Data utilised in these plots included the Benjamini-Hochberg adjusted $p$ value, the percentage of the genes from the RNA-seq datasets that aligned to this pathway and the fold enrichment, which was defined as the proportion of genes present in this list compared to the Spil RNA-Seq gene expression background.

Hierarchical clustering analysis. Hierarchical clustering was performed utilising 'gplots', 'dendextend' and 'colorspace' packages in $\mathrm{R}$ with code adapted from ${ }^{46,47}$, to produce a heatmap and dendrogram to better visualise the clusters. Complete markdown can be found in Supplementary Methods. FPKM values were $\log _{10}$ transformed, and z-scored (Pearson Correlation method) prior to clustering. Hierarchical clustering resulted in six distinct clusters which are highlighted in different colours in the dendrogram (Fig. 4).

Enrichment of association signal in IGAP GWAS data. As Spi1 has been implicated in the regulation of other $\mathrm{AD}$ risk gene expression, both RNA-Seq datasets were tested for enrichment of association with $\mathrm{AD}$ risk in the International Genomics of Alzheimer's Project (IGAP) GWAS dataset ${ }^{67}$. Firstly, the BioMart feature in Ensembl was used to convert mouse genes differentially expressed in the Spi1 knock-down and over-expression datasets into human orthologs ${ }^{68}$.

Gene sets were then determined for each Spi1 RNA-Seq dataset using $p$ value cut-offs between 0.05 and $1 \times 10^{-10}$. Direction of differential expression was not considered when defining gene sets. These gene sets (Supplementary Table 2) were tested for enrichment in the IGAP dataset using Multi-marker Analysis of GenoMic Annotation (MAGMA) analysis ${ }^{69}$.

Flow cytometric analysis of PU.1. Mixed glial cultures were harvested on day 21. Culture media was carefully removed and retained. Cells were detached by incubating with Accumax for $10-20 \mathrm{~min}$ at $37^{\circ} \mathrm{C}$, added to the appropriate culture media and centrifuged at $300 \times g$ for $7 \mathrm{~min}$. Approximately $2 \times 10^{5}$ cells per sample were fixed in $4 \%$ formalin solution (in DPBS) on ice for $30 \mathrm{~min}$. Formalin was removed by centrifugation $(300 \times g$ for $7 \mathrm{~min}$ ) and cells were permeabilised on ice in $90 \%$ ice cold methanol (v/v in PBS). Samples were centrifuged at $300 \times g$ for $7 \mathrm{~min}$ and the supernatant discarded. To ensure the methanol was completely removed an additional wash step using $500 \mu \mathrm{L}$ of wash solution $\left(0.5 \%\right.$ (w/v) BSA, $5 \mathrm{mM} \mathrm{EDTA}$ and $2 \mathrm{mM} \mathrm{NaN}_{3}$ in DPBS) and centrifugation at $300 \times g$ for $7 \mathrm{~min}$. The supernatant was discarded, and the cell pellets were then re-suspended in $50 \mu \mathrm{L}$ of block solution ( $5 \%(\mathrm{v} / \mathrm{v})$ filtered Rabbit Serum, $4 \mu \mathrm{g} / \mathrm{mL}$ Rat Anti-mouse Fc $\gamma$ RII/III 2.4G2 clone in wash solution) and incubated on ice for 20-30 min. Following the blocking step $50 \mu \mathrm{L}$ of CD11b, CD45 and PU.1 antibodies (listed below) were added and cells were incubated for 30-60 min on ice in the dark. The following antibodies were used in Flow cytometric experiments; Anti-CD11b FITC (56C, $8 \mu \mathrm{g} / \mathrm{mL}$, produced in house), Anti-CD11b

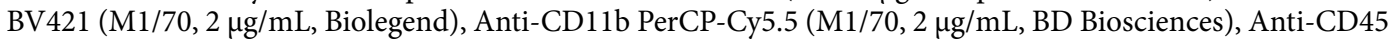
eFlour450 or PE-Cyanine7 conjugates (30-F11, $2 \mu \mathrm{g} / \mathrm{mL}$, eBioscience), Anti-PU.1 AF647 (7C2C34, $5 \mu \mathrm{g} / \mathrm{mL}$, Biolegend) and Rat IgG2a,k AF647 Isotype Control (RTK2758, $5 \mu \mathrm{g} / \mathrm{mL}$, Biolegend). 
Cells were washed with wash solution 3 times and centrifuged at $300 \times g$ for $7 \mathrm{~min}$. After the final wash the cell pellets were re-suspended in $500 \mu \mathrm{L}$ of wash solution and acquired on Attune NxT cytometer (Thermofisher). Data was analysed using FlowJo software (version 10; FlowJo LLC). Single colour and isotype controls were used as appropriate. Post data collection relative PU.1 protein levels were determined using the Median Fluorescent Intensity (MFI) values in the PU.1 antibody channel. First the isotype background signal from the same channel was subtracted. These values were then normalised between experiments by diving each value by the average MFI value (minus isotype) of the appropriate control sample.

Statistics and figures. Statistical analyses were performed using GraphPad PRISM 6 (version 3.07) and GraphPad PRISM 8 (version 8.4.3; both GraphPad Software, Inc.), unless otherwise stated. All statistical tests will be described as appropriate. $p$ values of $>0.05$ were taken as non-significant (ns). $p$ values of $\leq 0.05$ will be denoted with a single asterisk ${ }^{*}, p$ values of $\leq 0.01$ will be written as ${ }^{* *}, p$ values of $\leq 0.001$ by ${ }^{* *}$ and $p$ value of $\leq 0.0001$ as ${ }^{* * *}$. Figures were made using both GraphPad PRISM 6, GraphPad PRISM 8 (version 3.07 \& 8.4.3; GraphPad Software, Inc.) and R Studio (Version 1.2.5042 copyright 2009-2020 RStudio, Inc. ${ }^{39}$ ) with base R version 4.0.0 (2020-04-24, copyright 2020 The R Foundation for Statistical Computing $\left.{ }^{40}\right)$. The following R packages

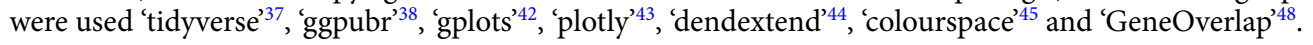

Received: 11 February 2021; Accepted: 7 July 2021

Published online: 22 July 2021

\section{References}

1. Prince, M. et al. Dementia UK: Second Edition - Overview 61 (Alzheimer's Society, 2014).

2. Jones, L. et al. Genetic evidence implicates the immune system and cholesterol metabolism in the aetiology of Alzheimer's disease. PLoS ONE 5(11), 13950 (2010).

3. Escott-Price, V. et al. Gene-wide analysis detects two new susceptibility genes for Alzheimer's disease. PLoS ONE 9(6), 94661 (2014).

4. Kunkle, B. W. et al. Genetic meta-analysis of diagnosed Alzheimer's disease identifies new risk loci and implicates A $\beta$, tau, immunity and lipid processing. Nat Genet. 51, 414-430 (2019).

5. Sims, R. et al. Rare coding variants in PLCG2, ABI3, and TREM2 implicate microglial-mediated innate immunity in Alzheimer's disease. Nat. Genet. 49(9), 1373-1384 (2017).

6. Gjoneska, E. et al. Conserved epigenomic signals in mice and humans reveal immune basis of Alzheimer's disease. Nature 518, 365-369 (2015).

7. Huang, K. et al. A common haplotype lowers SPI1 (PU.1) expression in myeloid cells and delays age at onset for Alzheimer's disease. Nat. Neurosci. 20, 1052-1061 (2017).

8. Tansey, K. E., Cameron, D. \& Hill, M. J. Genetic risk for Alzheimer's disease is concentrated in specific macrophage and microglial transcriptional networks. Genome Med. 10(14), 1-10 (2018).

9. Celada, A. et al. The transcription Factor PU.1 is involved in macrophage proliferation. J. Exp. Med. 184, 61-69 (1996).

10. Anderson, K. L. et al. Myeloid development is selectively disrupted in PU.1 null mice. Blood 91(10), 3702-3710 (1998).

11. Beers, D. R. et al. Wild-type microglia extend survival in PU.1 knockout mice with familial amyotrophic lateral sclerosis. PNAS 103(43), 16021-16026 (2006).

12. Scott, E. W., Simon, M. C., Anastasi, J. \& Singh, H. Requirement of transcription factor PU.1 in the development of multiple hematopoietic lineages. Science 265, 1573-1577 (1994).

13. McKercher, S. R. et al. Targeted disruption of the PU.1 gene results in multiple hematopoietic abnormalities. EMBO J. 15(20), 5647-5658 (1996).

14. DeKoter, R. P. \& Singh, H. Regulation of B lymphocyte and macrophage development by graded expression of PU.1. Science 288, 1439-1441 (2000).

15. Back, J., Dierich, A., Bronn, C., Kastner, P. \& Chan, S. PU.1 determines the self-renewal capacity of erythroid progenitor cells. Blood 103(10), 3615-3623 (2004).

16. Back, J., Allman, D., Chan, S. \& Kastner, P. Visualizing PU.1 activity during hematopoiesis. Exp. Hematol. 33, 395-402 (2005).

17. Hu, Z. et al. RUNX1 regulates corepressor interactions of PU.1. Blood 117(24), 6498-6508 (2011).

18. Jin, H. et al. Runxl regulates embryonic myeloid fate choice in zebrafish through a negative feedback loop inhibiting Pu.1 expression. Blood 119(22), 5239-5249 (2012).

19. Zarnegar, M. A., Chen, J. \& Rothenberg, E. V. Cell-type-specific activation and repression of PU.1 by a complex of discrete, functionally specialized cis-regulatory elements. Mol. Cell. Biol. 30(20), 4922-4939 (2010).

20. Li, Y. et al. Regulation of the PU. 1 gene by distal elements. Blood 98(10), 2958-2965 (2001).

21. Okuno, Y. et al. Potential autoregulation of transcription factor PU.1 by an upstream regulatory element. Mol. Cell. Biol. 25(7), 2832-2845 (2005).

22. Zarnegar, M. A. \& Rothenberg, E. V. Ikaros represses and activates PU.1 cell-type-specifically through the multifunctional Sfpil URE and a myeloid specific enhancer. Oncogene 25(31), 4647-4554 (2012).

23. Leddin, M. et al. Two distinct auto-regulatory loops operate at the PU.1 locus in B cells and myeloid cells. Blood 117(10), 2827-2838 (2011).

24. Kamath, M. B. et al. Dose-dependent repression of T-cell and natural killer cell genes by PU.1 enforces myeloid and B-cell identity. Leukemia 22, 1214-1225 (2008).

25. Lloberas, J., Soler, C. \& Celada, A. The key role of PU.1/SPI-1 in B cells, myeloid cells and macrophages. Immunol. Today 20(4), 184-189 (1999).

26. Pahl, H. L. et al. The proto-oncogene PU.1 regulates expression of the myeloid-specific CD11b promoter. J. Biol. Chem. 268(7), 5014-5020 (1993).

27. Anderson, K. L. et al. PU.1 is a lineage-specific regulator of tyrosine phosphatase CD45. J. Biol. Chem. 276(10), 7637-7642 (2001).

28. Heinz, S. et al. Simple combinations of lineage-determining transcription factors prime cis-regulatory elements required for macrophage and B cell identities. Mol. Cell 38, 576-589 (2010).

29. Ghisletti, S. et al. Identification and characterization of enhancers controlling the inflammatory gene expression program in macrophages. Immunity 32, 317-328 (2010).

30. Gosselin, D. et al. Environment drives selection and function of enhancers controlling tissue-specific macrophage identities. Cell 159, 1327-1340 (2014). 
31. Rustenhoven, J. et al. PU.1 regulates Alzheimer's disease-associated genes in primary human microglia. Mol. Neurodegener. 13, 44. https://doi.org/10.1186/s13024-018-0277-1 (2018).

32. Pimenova, A. A. et al. Alzheimer's-associated PU.1 expression levels regulate microglial inflammatory response. Neurobiol. Dis. 148, $105217(2021)$.

33. Olmos-Alonso, A. et al. Pharmacological targeting of CSF1R inhibits microglial proliferation and prevents the progression of Alzheimer's-like pathology. Brain 139, 891-907 (2016).

34. Dagher, N. N. et al. Colony-stimulating factor 1 receptor inhibition prevents microglial plaque association and improves cognition in 3xTg-AD mice. J. Neuroinflam. 12(139), 1-14 (2015).

35. Sosna, J. et al. Early long-term administration of the CSF1R inhibitor PLX3397 ablates microglia and reduces accumulation of intraneuronal amyloid, neuritic plaque deposition and pre-fibrillar oligomers in 5XFAD mouse model of Alzheimer's disease. Mol. Neurodegener. 13, 1-11 (2018).

36. Spangenberg, E. E. et al. Eliminating microglia in Alzheimer's mice prevents neuronal loss without modulating amyloid- $\beta$ pathology. Brain 139, 1265-1281 (2016).

37. Wickham, H. et al. Welcome to the Tidyverse. J. Open Source Softw. 4(43), 1686 (2019).

38. Kassambara, A. ggpubr: 'ggplot2' Based Publication Ready Plots. https://rpkgs.datanovia.com/ggpubr/index.html

39. RStudio Team. RStudio: Integrated Development for R. RStudio (2020).

40. R Core Team. R: A Language and Environment for Statistical Computing [Internet]. R Foundation for Statistical Computing; 2020. Available from: https://www.R-project.org/.

41. Smith, A. M. et al. The transcription factor PU.1 is critical for viability and function of human brain microglia. Glia 61, 929-942. https://doi.org/10.1002/glia.22486 (2013).

42. Warnes, G. R., Bolker, B., Bonebakker, L., Gentleman, R., Huber, W., Liaw, A. et al. gplots: Various R Programming Tools for Plotting Data [Internet]. 2020. Available from: https://cran.r-project.org/web/packages/gplots/index.html.

43. Plotly Technologies Inc. Collaborative Data Science. Plotly Technologies Inc. (2015).

44. Galili, T. dendextend: An R package for visualizing, adjusting and comparing trees of hierarchical clustering. Bioinformatics 31(22), 3718-3720 (2015).

45. Zeileis, A., Fisher, J. C., Hornik, K., Ihaka, R., McWhite, C. D., Murrell, P. et al. colorspace: A toolbox for manipulating and assessing colors and palettes. J. Stat. Softw. 96, 5-6 (2020).

46. Liu, Y. How to Draw Heatmap with Colorful Dendrogram [Internet]. Yang's Research Blog. 2018 [cited 2020 Apr 23]. p. 1. Available from: https://liuyanguu.github.io/post/2018/07/16/how-to-draw-heatmap-with-colorful-dendrogram/.

47. Brandon, Y. How to plot a Heatmap in Rstudio, the easy way [Internet]. 2019 [cited 2020 Apr 23]. Available from: https://www. youtube.com/watch?v=OWWHfXgRw3k.

48. Shen, L. Sinai ISoMaM. GeneOverlap: Test and visualize gene overlaps. R package version 1.28.0. https://doi.org/10.18129/B9. bioc.GeneOverlap (2021).

49. Zhang, D. E., Hetherington, C. J., Chen, H. M. \& Tenen, D. G. The macrophage transcription factor PU.1 directs tissue-specific expression of the macrophage colony-stimulating factor receptor. Mol. Cell. Biol. 14(1), 373-381 (1994).

50. Sierksma, A. et al. Novel Alzheimer risk genes determine the microglia response to amyloid- $\beta$ but not to TAU pathology. EMBO Mol. Med. 12(3), e10606 (2020).

51. Marecki, S., Riendeau, C. J., Liang, M. D. \& Fenton, M. J. PU.1 and multiple IFN regulatory factor proteins synergize to mediate transcriptional activation of the human IL-1 $\beta$ gene. J. Immunol. 166, 6892-6838 (2001).

52. Salih, D. A. et al. Genetic variability in response to amyloid beta deposition influences Alzheimer's disease risk. Brain Commun. 1(1), 1-13 (2019).

53. Schoggins, J. W. Interferon-stimulated genes: What do they all do?. Annu. Rev. Virol. 6(1), 567-584 (2019).

54. Smith, A. M. et al. Adult human glia, pericytes and meningeal fibroblasts respond similarly to IFNy but not to TGF $\beta 1$ or M-CSF. PLoS ONE 8(12), e80463 (2013).

55. Srinivasan, K. et al. Alzheimer's patient microglia exhibit enhanced aging and unique transcriptional activation. Cell Rep. 31(13), $107843(2020)$.

56. Butovsky, O. et al. Identification of a unique TGF- $\beta$-dependent molecular and functional signature in microglia. Nat. Neurosci. 17(1), 131-143 (2014).

57. Bohlen, C. J. et al. Diverse requirements for microglial survival, specification, and function revealed by defined-medium cultures. Neuron 94, 759-773 (2017).

58. Rosas, M. et al. The transcription factor Gata6 links tissue macrophage phenotype and proliferative renewal. Science 344, 645-648 (2014).

59. Ipseiz, N. et al. Effective in vivo gene modification in mouse tissue-resident peritoneal macrophages by intraperitoneal delivery of lentiviral vectors. Mol. Ther. Methods Clin. Dev. 16, 21-31 (2020).

60. Bolger, A. M., Lohse, M. \& Usadel, B. Trimmomatic: A flexible trimmer for Illumina sequence data. Bioinformatics 30(15), 21142120 (2014).

61. Simon, A. FastQC A quality control tool for high throughput sequence data [Internet]. Babraham Bioinformatics. Available from: http://www.bioinformatics.babraham.ac.uk/projects/fastqc/.

62. Dobin, A. et al. STAR: Ultrafast universal RNA-seq aligner. Bioinformatics 29(1), 15-21 (2013).

63. Liao, Y., Smyth, G. K. \& Shi, W. featureCounts: An efficient general purpose program for assigning sequence reads to genomic features. Bioinformatics 30(7), 923-930 (2014).

64. Ensembl FTP Download [Internet]. Available from: ftp://ftp.ensembl.org/pub.

65. Love, M. I., Huber, W. \& Anders, S. Moderated estimation of fold change and dispersion for RNA-seq data with DESeq2. Genome Biol. 15(12), 550 (2014).

66. Zheng, R. et al. Cistrome data browser: Expanded datasets and new tools for gene regulatory analysis. Nucleic Acids Res. 47(D1), D729-D735 (2019).

67. Lambert, J. C. et al. Meta-analysis of 74,046 individuals identifies 11 new susceptibility loci for Alzheimer's disease. Nat. Genet. 45(12), 1452-1458 (2013).

68. Herrero, J. How to get all the orthologous genes between two species [Internet]. Ensembl Blog. 2009 [cited 2018 Nov 20]. Available from: http://www.ensembl.info/2009/01/21/how-to-get-all-the-orthologous-genes-between-two-species/.

69. De Leeuw, C. A., Mooij, J. M., Heskes, T. \& Posthuma, D. MAGMA: Generalized gene-set analysis of GWAS data. PLoS Comput. Biol. 11(4), 1-19 (2015).

\section{Acknowledgements}

We would like to thank our animal facility staff for their care for the animals used in this study. We also thank the Cardiff University data clinic volunteers for their advice, and especially to thank Dr John Watkins for his guidance. We acknowledge Wales Gene Park and the Advanced Research Computing Team at Cardiff University, for performing Illumina sequencing and provision of high-performance computing infrastructure. This Project 
was funded by the MRC-Doctoral Training Programme Studentship. PRT is funded by a UK Dementia Research Institute Professorship and a Wellcome Trust Investigator Award (107964/Z/15/Z).

\section{Author contributions}

R.J. conducted the work; R.A., P.H. and M.H. assisted with data analysis and interpretation; R.J. and P.R.T. conceived and designed the study and wrote the manuscript; All authors commented on and approved the manuscript.

\section{Competing interests}

M.H. is now employed by Vertex Pharmaceuticals. The other authors declare that there are no other competing interests.

\section{Additional information}

Supplementary Information The online version contains supplementary material available at https://doi.org/ 10.1038/s41598-021-94324-Z.

Correspondence and requests for materials should be addressed to P.R.T.

Reprints and permissions information is available at www.nature.com/reprints.

Publisher's note Springer Nature remains neutral with regard to jurisdictional claims in published maps and institutional affiliations.

(c) (i) Open Access This article is licensed under a Creative Commons Attribution 4.0 International License, which permits use, sharing, adaptation, distribution and reproduction in any medium or format, as long as you give appropriate credit to the original author(s) and the source, provide a link to the Creative Commons licence, and indicate if changes were made. The images or other third party material in this article are included in the article's Creative Commons licence, unless indicated otherwise in a credit line to the material. If material is not included in the article's Creative Commons licence and your intended use is not permitted by statutory regulation or exceeds the permitted use, you will need to obtain permission directly from the copyright holder. To view a copy of this licence, visit http://creativecommons.org/licenses/by/4.0/.

(C) The Author(s) 2021 